\title{
Caracterização demográfica de usuários do ambulatório de saúde mental de Santa Maria - RS
}

\author{
Aline Sarturi Ponte ${ }^{1}$ Ana Cristina Von Bock Bolli2 Maria Saleti Lock Vogt ${ }^{3}$ Miriam Cabrera Corvelo Delboni ${ }^{4}$ Rosimara \\ Adilia Piovezan ${ }^{5}$
}

\begin{abstract}
RESUMO
Objetivo - caracterizar demograficamente os usuários do ambulatório de saúde mental de Santa Maria/RS. Metodologia - é uma pesquisa é de caráter descritivo, com análise quantitativa, coletaram-se dados de 708 prontuários. Resultados - observou-se a predominância do sexo feminino (74,3\%); quanto à situação civil 42,7\% são casados; a escolaridade da maioria (62,3\%) dos sujeitos compreende o ensino fundamental completo; $51,7 \%$ dos usuários são adultos ( 40 - 65 anos de idade); o diagnóstico mais citado está entre o F30 - F39 em 53\%; o comprometimento funcional variou entre pouco e muito, alcançando quase a totalidade dos indivíduos. 0 uso de medicamentos é predominante entre os pesquisados. Discussão - a avaliação de estudos demográficos é necessária para o conhecimento do perfil dos usuários de um serviço com o foco de traçar objetivos reais de intervenção para uma determinada região. Conclusão - o estudo revelou significativos apontamentos para o delineamento de atenção a saúde no que se refere as pessoas com transtornos psíquicos.
\end{abstract}

Descritores: Demografia; Perfil de Saúde; Saúde Mental.

\section{Demographic characteristics of users of mental health clinic in Santa Maria - RS}

\begin{abstract}
Objective - characterize demographically users of mental health clinic in Santa Maria / RS. Methodology - is a research is descriptive, and quantitative analysis, we collected data from 708 records. Results - There was a predominance of females (74.3\%); regarding the civil status $42.7 \%$ are married; the education of the majority (62.3\%) of the subjects comprising the complete primary education; $51.7 \%$ of users are adults (40-65 years old); the most cited diagnosis is between F30 - F39 in 53\%; functional impairment ranged between low and high, reaching almost all individuals. The use of drugs is prevalent among those surveyed. Discussion - a review of population studies is necessary for knowledge about the users of a service with the focus of outlining real goals of intervention for a certain region. Conclusion - the study revealed significant trends for the design of health care regarding people with mental disorders.
\end{abstract}

Descriptors: Demography; Health Profile; Mental Health.

\footnotetext{
${ }^{1}$ Mestranda em Distúrbios da Comunicação Humana na Universidade Federal de Santa Maria (UFSM) Santa Maria, RS, Brasil.

${ }^{2}$ Mestre em Psicologia pela Universidade Federal do Rio Grande do Sul (UFRGS), Porto Alegre, RS, Brasil.

${ }^{3}$ Doutora em Ciências da Saúde pela Universidade de Brasília (UnB), Brasília, DF, Brasil.

${ }^{4}$ Doutoranda em Desenvolvimento Regional na Universidade de Santa Cruz do Sul (UNISC), Santa Cruz, RS, Brasil.

${ }^{5}$ Terapeuta Ocupacional pelo Centro Universitário Franciscano (UNIFRA), Santa Maria, RS, Brasil.
} 


\section{Introdução}

Nos anos de 1970 o Brasil foi marcado pelo movimento sanitário cujas discussões favoreciam mudanças nos modelos de atenção e gestão nas práticas de saúde, na busca de traçar novos rumos para saúde brasileira. O processo de Reforma Psiquiátrica no Brasil esteve aliado a este movimento com propósito de traçar estratégias inclusivas para atenção em saúde mental em nosso país. ${ }^{1}$

O processo da Reforma Psiquiátrica ocorreu durante a ditadura militar, época em que a medicalização era o modelo básico de intervenção. 0 poder centralizador do hospital psiquiátrico e o elevado índice de internações passaram a ser consideradas as causas estruturais das condições desumanas a que eram submetidos os pacientes psiquiátricos ${ }^{1}$.

Os caminhos delineados a partir dos fatos ocorridos nesta década desencadearam na saúde mental a necessidade de intervenções que teve como propósito a inserção da pessoa com sofrimento psíquico na comunidade. Tais mudanças deram início ao desenvolvimento de terapia de grupos com pacientes, porém, essa atenção não ficou voltada somente a estes, pois os familiares também receberam suporte terapêutico com a criação dos grupos familiares. ${ }^{2}$

Avançou a visão de tratamento da pessoa com sofrimento psíquico, tendo como proposta o deslocamento da assistência segregadora no sistema asilar e em hospitais psiquiátricos, substituída pelos programas que incentivaram a volta do paciente para o convívio familiar, as residências terapêuticas e as internações em hospital geral, nas unidades psiquiátricas, porém, por um período de curta duração. Criaram-se novas modalidades de serviços para atender esta população, como Centro de Assistência Psicossocial (CAPs), Hospital-Dia, Ambulatórios de Saúde Mental, dando ênfase para o tratamento nos serviços terapêuticos da comunidade ${ }^{2}$. Esta visão diferenciada contribuiu para evolução do tratamento, e também auxiliou no processo de inclusão destas pessoas ao mesmo meio social ao qual estão inseridos seus familiares e amigos. ${ }^{3}$

A Política Nacional de Saúde Mental, que norteia atualmente a Reforma Psiquiátrica, estimula práticas pautadas no território e articuladas em uma rede ampliada de serviços de saúde. Mas as lacunas ainda parecem ser grandes entre 0 que essas diretrizes propõem e o que se observa na realidade concreta. Ademais, as concepções e práticas em saúde mental por ventura existentes no nível básico de atenção à saúde nem sempre condizem com o esperado por parte dos que formulam a Reforma Psiquiátrica Brasileira, gerando por vezes questionamentos quanto à sua real contribuição no sentido de avançar na reinserção social do portador de transtornos mentais e na desestigmatização e cuidado efetivo dessas pessoas. ${ }^{4}$

A Organização Mundial da Saúde aponta que os transtornos mentais de cerca de 450 milhões de pessoas ainda estão longe de receberem a mesma relevância dada à saúde física, sobretudo nos países em desenvolvimento. Frente aos dados apresentados pela OMS considera-se relevante o desenvolvimento de estudos na área de saúde mental, assim como a interface entre saúde coletiva e saúde mental, pois "tal vinculação permitiria o desenvolvimento de investigações na interface entre saúde coletiva e saúde mental, que permanece ainda um território quase inexplorado, potencialmente uma área emergente de máxima relevância social" (p.1054). ${ }^{6}$

As discussões sobre saúde mental não se restringem à cura das doenças ou a sua prevenção, mas na prática de recursos que tenham como resultados melhores condições de saúde para a população. ${ }^{7}$ Não interessando somente a ausência de doenças, mas o desenvolvimento integral das pessoas e da comunidade. A ênfase, então, na saúde mental, desloca-se da doença à saúde e à observação de como os seres humanos vivem em seu cotidiano. ${ }^{8}$ Então conhecer a população de usuários do Ambulatório de Saúde Mental de Santa Maria/RS proporcionará observar as necessidades dos usuários e do próprio serviço, sendo assim poderá contribuir para uma avaliação do serviço e capacitação pessoal para atuar junto a equipe e aos usuários do mesmo. ${ }^{6}$

Este estudo tem como base o seguinte objetivo, apresentar a caracterização demográfica dos usuários de um ambulatório de saúde mental do município de Santa Maria/RS.

\section{Método}

Desenvolveu-se uma pesquisa descritiva, com análise quantitativa, realizada pelo Programa de Educação pelo Trabalho para a Saúde (PET-Saúde), edital 2010 - 2012, vinculado às instituições Centro Universitário Franciscano (UNIFRA) e Universidade Federal de Santa Maria (UFSM) e Ambulatório de Saúde Mental do Município de Santa Maria. 
A população deste estudo compreendeu todas as pessoas agendadas para consultas com os psiquiatras do serviço, durante os meses de junho a setembro de 2011. Foram acessados somente os prontuários daqueles que compareceram às consultas. Os dados foram registrados em uma ficha que continha as informações quanto ao sexo, idade (classificados em adolescente -12 aos 20 anos, adulto jovens -20 aos 40 anos, adultos -40 aos 65 anos, idosos - 65 anos em diante) 9 , ocupação, procedência, diagnósticos de doenças (agrupados por grupos de enfermidades, segundo a Classificação do Código Internacional de Doenças-10 - CID-10) (Figura 01)10, o comprometimento funcional, o uso de medicação, e encaminhamentos terapêuticos.

Figura 1 - Categorização das doenças segundo CID -10 referentes a transtornos psíquicos

\begin{tabular}{|c|c|}
\hline $\mathrm{F} 00-\mathrm{F} 09$ & $\begin{array}{l}\text { Transtornos mentais orgânicos inclusive os sintomáticos (Alzheimer, } \\
\text { Demência na doença de Parkinson, Demência na doença de Pick). }\end{array}$ \\
\hline $\mathrm{F} 10-\mathrm{F} 19$ & $\begin{array}{l}\text { Transtornos mentais e de comportamento decorrentes, do uso de } \\
\text { substâncias psicoativas (Transtornos mentais e de comportamento } \\
\text { devidos ao uso de álcool, drogas licitas e ilícitas, sedativos, tabaco, } \\
\text { substâncias estimulantes). }\end{array}$ \\
\hline $\mathrm{F} 20-\mathrm{F} 29$ & Esquizofrenia, distúrbios esquizotípicos e delirantes. \\
\hline $\mathrm{F} 30-\mathrm{F} 39$ & $\begin{array}{c}\text { Transtornos do humor (afetivos) (Episódio maníaco, Transtorno afetivo } \\
\text { bipolar, Episódio depressivo, Transtorno depressivo recorrente, transtornos } \\
\text { persistentes do humor). }\end{array}$ \\
\hline $\mathrm{F} 40-\mathrm{F} 49$ & $\begin{array}{l}\text { Transtornos neuróticos, relacionados ao estresse e somatoformes } \\
\text { (Transtornos fóbico-ansiosos, obsessivo-compulsivo, dissociativos, } \\
\text { Reação a estresse grave e transtornos da adaptação). }\end{array}$ \\
\hline $\mathrm{F} 50-\mathrm{F} 59$ & $\begin{array}{l}\text { Síndromes comportamentais associadas a distúrbios fisiológicos e fatores } \\
\text { físicos (Transtornos Alimentares, não-orgânicos o sono, Disfunção sexual } \\
\text { não causada por transtorno ou doença orgânica, Abuso de substâncias } \\
\text { que não produzem dependência). }\end{array}$ \\
\hline $\mathrm{F} 60-\mathrm{F} 69$ & $\begin{array}{c}\text { Transtornos de personalidade e de comportamento em adulto (Transtornos } \\
\text { específicos de personalidade, de personalidade mistos e outros, de } \\
\text { hábitos e impulsos, da identidade sexual, da preferência sexual) }\end{array}$ \\
\hline $\mathrm{F} 70-\mathrm{F} 79$ & $\begin{array}{l}\text { Retardo mental (Retardo mental leve, moderado, grave, profundo, Outro } \\
\text { retardo mental, Retardo mental, não especificado). }\end{array}$ \\
\hline F80 - F89 & $\begin{array}{l}\text { Transtornos do desenvolvimento psicológico (Transtornos específicos } \\
\text { do desenvolvimento da fala e da linguagem, das habilidades escolares, } \\
\text { ranstorno específico do desenvolvimento da função motora, mistos do } \\
\text { desenvolvimento, globais do desenvolvimento) }\end{array}$ \\
\hline F90 - F99 & $\begin{array}{l}\text { Transtornos emocionais e de comportamento com início ocorrendo } \\
\text { habitualmente na infância ou adolescência e transtorno mental não } \\
\text { especificado (Transtornos hipercinéticos, de conduta, mistos de conduta } \\
\text { e das emoções, emocionais com início especificamente na infância, } \\
\text { do funcionamento social com início especificamente na infância ou na } \\
\text { adolescência) }\end{array}$ \\
\hline
\end{tabular}

Adotou-se a denominação "múltiplos" para os pacientes que apresentavam duas ou mais patologias descritas no prontuário. Para os pacientes novos que estavam em processo de investigação da doença utilizou-se o termo "em avaliação".

Os dados foram armazenados no Microsoft Office Excel 2007 e a análise foi processada no Software SPSS versão 13.0. Ao analisar as variáveis quantitativas (idade e tempo da doença) verificou-se que as mesmas não possuíam distribuição normal, com isso, foi utilizada a análise estatística não-paramétrica. Foram realizados os testes Shapiro - Wilk's e Kolmogorov-Smirnov. ${ }^{11,12}$ para os dados de normalidade. Para verificar a existência de associação entre as variáveis foi realizado o teste de Qui-quadrado (teste de independência) e para outros cruzamentos com variáveis quantitativas e qualitativas utilizou-se o teste $U$ de Mann - Whitney e Kruskall - Wallis. A significância estatística utilizada na realização dos testes foi de $5 \%(p \leq 0,005)$. 
O projeto de pesquisa foi aprovado pelo Comitê de Ética e Pesquisa da Universidade Federal Santa Maria - UFSM, sendo aprovado em 14/06/2011, com processo de encaminhamento $N^{0}$ 23081.008032/2011-67, conforme prevê a Resolução 196/96 e 251/97 do Conselho Nacional de Saúde - CNS, que visa fundamentalmente normatizar as pesquisas que envolvem seres humanos.

Os resultados, a seguir, expressam uma caracterização dos usuários que receberam atendimento psiquiátrico ambulatorial e frequentaram o serviço no período em estudo.

\section{Resultados}

Foram analisados 708 prontuários dos usuários do Ambulatório de Saúde Mental de Santa Maria/RS e a partir dos dados coletados traçou-se um perfil da população assistida neste serviço (Tabela 1). Do total de prontuários $25,7 \%$ correspondiam a sujeitos do sexo masculino e $74,3 \%$ correspondiam ao sexo feminino. Dentre os prontuários analisados observou-se que $42,7 \%$ os sujeitos casados apresentavam alguma patologia psiquiátrica.

Ao levantar dos dados referentes a escolaridade, contatou-se um baixo nível escolar, pois a maioria dos usuários do serviço apresentam o Ensino Fundamental (62,3\%) como base escolar. Observou-se que mesmo com tantos programas voltados a educação algumas pessoas ainda não tem acesso a esta, pois 1,8\% dos participantes eram Analfabetos. $E$ quanto aos outros níveis escolares, o Ensino Médio representou 24,3\% e o Ensino Superior 0,7\% dos usuários. Alguns prontuários estavam incompletos representando $10,9 \%$ dos mesmos.

$O$ período etário de maior concentração neste estudo foi a idade adulta, entre 40 e 65 anos $(51,7 \%)$ e a terceira idade, dos 65 em diante $(24,2 \%)$ o que parece influenciar no computo de doenças mentais. Os adultos jovens (20 a 40 anos) representam $23,6 \%$ e os adolescentes $0,6 \%$ dos dados coletados.

Tabela 1 - Caracterização dos usuários de um ambulatório de saúde mental/CAPS em um município do RS ( $n=708)$.

\begin{tabular}{|c|c|c|}
\hline Variáveis & Frequência (n) & Percentual (\%) \\
\hline \multicolumn{3}{|l|}{ Sexo } \\
\hline Feminino & 526 & 74.3 \\
\hline Masculino & 182 & 25.7 \\
\hline \multicolumn{3}{|l|}{ Situação Civil } \\
\hline Casado & 302 & 42.7 \\
\hline Divorciado & 108 & 15.3 \\
\hline Solteiro & 196 & 27.7 \\
\hline Viúvo & 43 & 6.1 \\
\hline Não respondeu & 59 & 8.3 \\
\hline \multicolumn{3}{|l|}{ Escolaridade } \\
\hline Analfabeto & 13 & 1.8 \\
\hline Ensino Fundamental & 441 & 62.3 \\
\hline Ensino Médio & 172 & 24.3 \\
\hline Ensino Superior & 5 & 0.7 \\
\hline Não Respondeu & 77 & 10.9 \\
\hline \multicolumn{3}{|l|}{ Período etário } \\
\hline Adolescência (12 a 20 anos) & 4 & 0,6 \\
\hline Adulto Jovem (20 a 40 anos) & 167 & 23,6 \\
\hline Adulto (40 a 65 anos) & 366 & 51,7 \\
\hline Idoso(65 anos em diante) & 171 & 24,2 \\
\hline
\end{tabular}




\begin{tabular}{|c|c|c|}
\hline \multicolumn{3}{|l|}{ Diagnóstico } \\
\hline F00 - F09 & 1 & 0.1 \\
\hline F10 - F19 & 5 & 0.7 \\
\hline F20 - F29 & 39 & 5.5 \\
\hline F30 - F39 & 375 & 53.0 \\
\hline F40 - F49 & 94 & 13.3 \\
\hline F50 - F59 & 4 & 0.6 \\
\hline F60 - F69 & 20 & 2.8 \\
\hline F70 - F79 & 26 & 3.7 \\
\hline F 90 - F99 & 2 & 0.3 \\
\hline Múltiplos 28 & 115 & 16.2 \\
\hline Em avaliação & 27 & 3.8 \\
\hline \multicolumn{3}{|l|}{ Comprometimento Funcional } \\
\hline Com interferência & 302 & 42.7 \\
\hline Com pouco & 374 & 52.8 \\
\hline Nenhum & 32 & 4.5 \\
\hline \multicolumn{3}{|l|}{ Uso de medicamento } \\
\hline Não & 2 & 0.3 \\
\hline Sim & 706 & 99.7 \\
\hline
\end{tabular}

No que se refere aos dados de saúde, o diagnóstico mais decorrente está entre o F30 - F39 (53\%), que compreende as patologias descritas como Transtornos do humor (afetivos), Episódio maníaco, Transtorno afetivo bipolar, Episódio depressivo, Transtorno depressivo recorrente, transtornos persistentes do humor. Os sujeitos que apresentavam dois ou mais diagnósticos em seu prontuário apresentam-se na Tabela 1 como Múltiplos estes sujeitos representaram 16,2\% dos sujeitos. Os Transtornos neuróticos, relacionados ao estresse e somatoformes (F40 - F39) representado 53\% dos usuários.

Além destes são atendidos também os Transtornos Esquizofrênicos (5,5\%), o Retardo Mental (3,7\%), Transtornos de personalidade e de comportamento em adultos (2,8\%), Transtornos mentais e de comportamento decorrentes, do uso de substâncias psicoativas $(0,7 \%)$, Síndromes comportamentais associadas a distúrbios fisiológicos e fatores físicos $(0,6 \%)$, Transtornos emocionais e de comportamento com início ocorrendo habitualmente na infância ou adolescência e transtorno mental não especificado $(0,3 \%)$, Transtornos mentais orgânicos inclusive os sintomáticos $(0,1 \%)$, mas ainda encontravam-se em avaliação 3,8\% dos sujeitos atendidos pelo ambulatório.

Quanto à condição de funcionalidade dos sujeitos variou entre pouca interferência e muita interferência em quase a totalidade de $95,5 \%$. Verificou-se que há o predomínio de medicalização em $99,7 \%$ dos casos. Notou-se que e a utilização de medicamentos é a principal prática terapêutica na rede pública de saúde do município, já que não se identificou encaminhamentos a outros tratamentos terapêuticos nos prontuários avaliados.

Quando analisado a respeito do diagnóstico em relação ao sexo a associação revelou significância $(p<0,0001)$ com predomínio no sexo feminino e o diagnostico corresponde ao F30 - F39 (Síndromes comportamentais associadas a distúrbios fisiológicos e fatores físicos) (Tabela 2). Já os diagnósticos associados ao sexo masculino são os transtornos mentais e de comportamento decorrentes do uso de substâncias psicoativas (F10 - F19), esquizofrenia, distúrbios esquizotípicos e delirantes (F20 - F29), retardo mental (F70 - F79), transtornos emocionais e de comportamento com início ocorrendo habitualmente na infância ou adolescência e transtorno mental não especificado (F90- F99) e múltiplos. 
Tabela 2 - Resultados da correlação entre as variáveis diagnóstico e sexo

\begin{tabular}{|c|c|c|c|c|c|}
\hline \multirow[t]{3}{*}{ Diagnóstico } & \multicolumn{4}{|c|}{ Grupos } & \multirow[t]{3}{*}{ P-valor } \\
\hline & \multicolumn{2}{|c|}{ Feminino } & \multicolumn{2}{|c|}{ Masculino } & \\
\hline & $\mathrm{N}$ & $\%$ & $\mathrm{~N}$ & $\%$ & \\
\hline F00 - F09 & 1 & $(0,2)$ & 0 & $(0,0)$ & \multirow{11}{*}{$<0,0001$} \\
\hline F10 - F19 & 1 & $(0,2)$ & 4 & $(2,2)$ & \\
\hline F20 - F29 & 20 & $(3,8)$ & 19 & $(10,4)$ & \\
\hline F30 - F39 & 302 & $(57,4)$ & 73 & $(40,1)$ & \\
\hline F40 - F49 & 75 & $(14,3)$ & 19 & $(10,4)$ & \\
\hline F50 - F59 & 3 & $(0,6)$ & 1 & $(0,5)$ & \\
\hline F60 - F69 & 13 & $(2,5)$ & 7 & $(3,8)$ & \\
\hline F70 - F79 & 12 & $(2,3)$ & 14 & $(7,7)$ & \\
\hline F90 - F99 & 0 & $(0,0)$ & 2 & $(1,1)$ & \\
\hline Múltiplos & 77 & $(14,6)$ & 38 & $(20,9)$ & \\
\hline Em avaliação & 22 & $(4,2)$ & 5 & $(2,7)$ & \\
\hline
\end{tabular}

Pode-se obervar na tabela 2 que existe associação entre o diagnóstico e a idade em classes $((p<0,0001)$. Os diagnósticos associados aos usuários na adolescência são os por transtornos emocionais e os de comportamento com início ocorrendo habitualmente na infância ou adolescência e o transtorno mental não especificado ( $F 90$ - $F$ 99). Os diagnósticos associados aos pacientes adultos jovens são múltiplos, visto que pessoas com sofrimento psíquico que apresentam duas ou mais patologias associadas. Os diagnósticos associados às pessoas idosas são os transtornos do humor (afetivos) (F 30 - F 39) (Tabela 3).

Tabela 3 - Diagnóstico x Idade

\begin{tabular}{|c|c|c|c|c|c|c|c|c|c|}
\hline \multirow{3}{*}{ Diagnósticos } & \multicolumn{8}{|c|}{ Idade por classe } & \multirow[b]{2}{*}{ p-valor } \\
\hline & \multicolumn{2}{|c|}{ Adolescência } & \multicolumn{2}{|c|}{ Adulto Jovem } & \multicolumn{2}{|c|}{ Meia idade } & \multicolumn{2}{|c|}{ Terceira Idade } & \\
\hline & $\mathrm{N}$ & $\%$ & $\mathrm{~N}$ & $\%$ & $\mathrm{~N}$ & $\%$ & $\mathrm{~N}$ & $\%$ & \multirow{12}{*}{$<0,0001$} \\
\hline F00 - F09 & 0 & (0) & 0 & (0) & 0 & (0) & 1 & $(0,6)$ & \\
\hline F 10 - F19 & 0 & (0) & 1 & $(0,6)$ & 3 & $(0,8)$ & 1 & $(0,6)$ & \\
\hline F20 - F29 & 0 & (0) & 10 & $(6,0)$ & 15 & $(4,1)$ & 14 & $(8,2)$ & \\
\hline F $30-F 39$ & 1 & $(25,0)$ & 72 & $(43,1)$ & 198 & $(54,1)$ & 104 & $(60,8)$ & \\
\hline F 40 - F49 & 1 & $(25,0)$ & 23 & $(13,8)$ & 46 & $(12,6)$ & 24 & $(14,0)$ & \\
\hline F 50 - F59 & 0 & (0) & 3 & $(1,8)$ & 1 & $(0,3)$ & 0 & (0) & \\
\hline F60 - F69 & 0 & (0) & 7 & $(4,2)$ & 10 & $(2,7)$ & 3 & $(1,8)$ & \\
\hline F70 - F79 & 0 & (0) & 15 & $(9,0)$ & 9 & $(2,5)$ & 2 & $(1,2)$ & \\
\hline F 90 - F99 & 2 & $(50,0)$ & 0 & (0) & 0 & (0) & 0 & (0) & \\
\hline Múltiplos & 0 & $(0)$ & 32 & $(19,2)$ & 70 & $(19,1)$ & 13 & $(7,6)$ & \\
\hline Em avaliação & 0 & (0) & 4 & $(2,4)$ & 14 & $(3,8)$ & 9 & $(5,3)$ & \\
\hline
\end{tabular}

O ambulatório de saúde mental presta serviço a todas as regiões administrativas do município de Santa Maria/RS como representado na figura 2. A maior demanda procede da região oeste $(36,2 \%)$, seguida pelas regiões, centro com $15,3 \%$, norte $(14,7 \%)$, sul com $8,1 \%$, leste $(7,3 \%)$, nordeste $(6,8 \%)$, centro oeste $(4,2 \%)$, os distritos rurais com $3,7 \%$ e centro leste com 3,2\% dos usuários. Este serviço também atende pessoas encaminhadas de municípios vizinhos (0,6\%). 


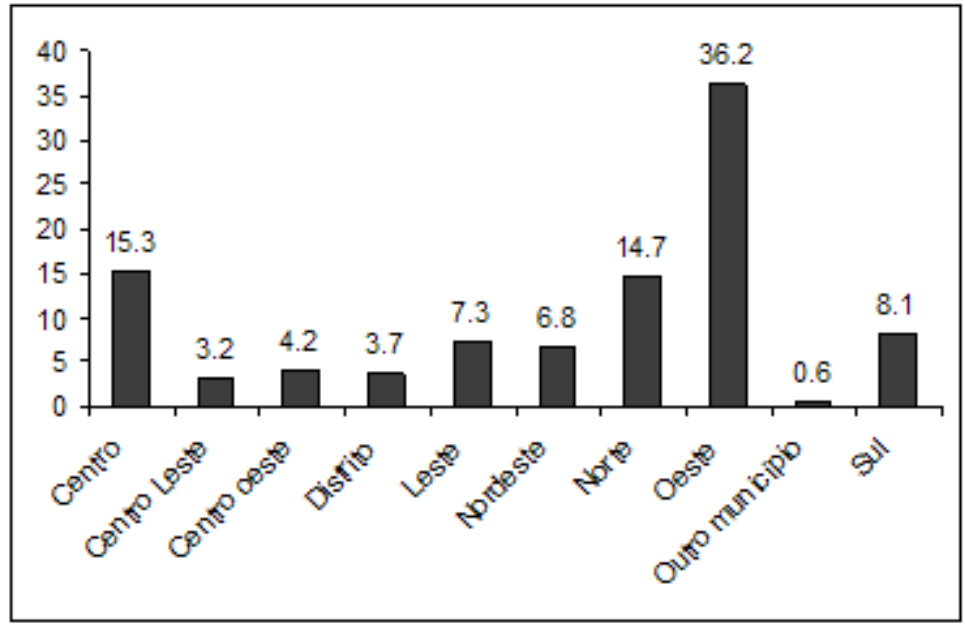

Figura 2 - Distribuição dos usuários segundo a região de procedência.

Observa-se um grande interesse na compreensão e identificação dos quadros clínicos e também no delineamento de estudos voltados para 0 atendimento dos casos. Entretanto, mesmo considerando esses aspectos, a produção nacional em saúde mental necessita de maior investimento nos objetivos de intervenção e prevenção.

\section{Discussões}

Ao analisar os dados coletados no ambulatório de saúde mental de Santa Maria/RS observou-se predominância do sexo feminino em sua maioria adultos, faixa etária que corresponde dos 40 aos 65 anos de idade (Tabela 1). "Os estudos de base populacional realizados em países ocidentais têm mostrado que cerca de $35 \%$ da população geral adulta não institucionalizada apresenta algum transtorno mental ao longo da vida" (p.44). ${ }^{13}$

$\mathrm{Na}$ idade adulta (40 e 65 anos) surgem inúmeras diferenças entre homens e mulheres em relação aos transtornos mentais. É uma fase marcada por transformações, as pessoas deparam-se com diferentes situações potencialmente estressoras, desemprego, necessidade de manutenção do emprego, criação dos filhos, o relacionamento enquanto casal relações interpessoais, preparação para aposentadoria e a própria aposentadoria, entre outros que podem ocorrer neste período, estão associados ao aparecimento e/ou à maior severidade em grande parte dos distúrbios psiquiátricos. ${ }^{14}$

Ao analisar a amostra total observou-se que diagnóstico predominante no serviço é o F 30 - F39 (Tabela 1), mas cruzarmos os dados diagnostico e sexo (Tabela 2) observa-se que no sexo feminino o diagnostico predominantes é o F30 - F39. A mulher apresenta vulnerabilidade acentuada a sintomas ansiosos e depressivos, principalmente associados ao período reprodutivo. ${ }^{15}$ Estudos apontam que a dupla jornada de trabalho (casa e trabalho) pode ser um fator contribuinte para o desencadeamento de patologias das mais diversificadas, além disso, os quadros depressivos têm representado o terceiro problema de saúde em mulheres nos países desenvolvidos e o quinto em países subdesenvolvidos, depois de causas maternas e de algumas doenças transmissíveis. ${ }^{7}$

Já no sexo masculino os diagnósticos apresenta-se distribuídos entre F10 - F19, F20 - 29, F 70 - F 79, F90 - F99 e diagnósticos múltiplos (Tabela 2). Os homens apresentam uma maior prevalência de transtornos associados ao uso de substâncias psicoativas, incluindo álcool, transtornos de personalidade, esquizotípica, transtornos do controle de impulsos e de déficit de atenção e hiperatividade na infância e na vida adulta ${ }^{13}$.

A relação do diagnóstico e da idade (Tabela 3 ) demonstrou que os adolescentes são mais acometidos pelos transtornos de conduta, atenção e hiperatividade e transtornos emocionais são os mais comuns entre os adolescentes (12 aos 20 anos. A discussão no meio acadêmico e clínico sobre estas patologias são importantes, porque resultam em sofrimento na adolescência e àqueles com quem convivem e, também, porque interferem no desenvolvimento psicossocial e educacional, podendo gerar problemas psiquiátricos e problemas no relacionamento interpessoal na vida adulta. ${ }^{16}$ Frente a estes dados observa-se a necessidade da criação de estratégias de atenção a saúde mental, visa-se a importância da implantação e implementação de serviços de saúde mental comunitários especializados na atenção a adolescência.

"A prevalência de transtornos mentais em adultos jovens (20 aos 40 anos) é alta, podendo chegar a 25 - 40\%, possivelmente porque nesta fase a maioria dos transtornos psiquiátricos ocorre em frequências mais elevadas na 
população." (p. 27) ${ }^{14}$ Um estudo realizado em Pelotas/RS aponta, a pobreza, desemprego, tabagismo, doenças crônicas, indivíduos com baixo apoio social ou que sofreram eventos estressantes, indivíduos com baixa escolaridade ou cujas mães tinham baixa escolaridade, que nasceram com baixo peso, como fatores que contribuem para o surgimento de transtornos mentais em adultos. ${ }^{18} \mathrm{Ao}$ atingir a idade adulta (40 aos 65 anos) estes "deparam-se com diferentes situações potencialmente estressoras como a criação dos filhos, o relacionamento enquanto casal, relações interpessoais, necessidade de manutenção do emprego e a própria aposentadoria." (p.71) ${ }^{17}$

O baixo nível escolar é um fator que é citado como um causador de transtornos mentais a longo prazo. ${ }^{15}$ Uma pesquisa realizada por Lopes, Faerstein, Chor (2003) no município de Pelotas/RS demonstra que o baixo nível de educação, que foi acima de $50 \%$, os autores desde estudo sugerem que se a educação superior fosse disponibilizada igualmente para toda a população este resultado poderia reduzir-se a metade. ${ }^{18}$

Os dados apesentados sobre escolaridade na Tabela 1 corroboram com os estudo citados anteriormente, demostrando que o baixo nível escolar pode ser um fator contribuinte para o desenvolvimento de transtornos mentais. Um estudo realizado em Pelotas/RS aponta, baixo apoio social ou que sofreram eventos estressantes, indivíduos com baixa escolaridade ou cujas mães tinham baixa escolaridade, que nasceram com baixo peso, como fatores que contribuem para o surgimento de transtornos mentais em adultos. ${ }^{19}$

A funcionalidade "indica os aspectos positivos da interação entre um indivíduo e os seus fatores contextuais (ambientais e pessoais)." (p. 165) ${ }^{20}$ Os transtornos mentais em geral causam considerável comprometimento funcional, assim representando um imenso desafio para o sujeito, seus familiares e cuidadores. ${ }^{20,}{ }^{21}$ Observa-se (Tabela 1) nos dados coletados que o transtorno mental contribui para um déficit na funcionalidade dos sujeito acometidos por transtornos mentais, resultando em limitações cotidianas, ou seja comprometimento em suas atividades de vida diária, atividades instrumentais de vida diária, atividades de laborais e de lazer. 0 impacto destas patologias acarreta, prejuízos na funcionalidade e diminuição da qualidade de vida de seus portadores. ${ }^{21}$

Os medicamentos são o principal foco de intervenção do ambulatório (Tabela 1). Diante desta realidade observa-se necessidade de conscientização da equipe médica para o encaminhamento destes usuários a outros de tratamentos como psicológico, terapêutico ocupacional e psicopedagógico para os adolescentes. A prescrição e o consumo excessivo de medicamentos psicotrópicos são fenômenos presentes no cotidiano dos serviços públicos de saúde, mas não só em nossa realidade. Tal consumo está relacionado ao papel exercido pela indústria farmacêutica na atenção à saúde, à força do modelo biomédico ancorado na biologia do processo saúde/doença, às concepções e práticas de saúde, bem como às demandas de felicidade características da modernidade, concretizáveis por meio dos medicamentos, especialmente os psicotrópicos.

A administração medicamentosa durante o período de tratamento apresenta contribuições para o sujeito, mas as formas de administração destes medicamentos são fatores preocupantes para órgãos públicos envolvidos com a saúde, tanto internacionais, como a OMS e Organização Pan-Americana da Saúde (Opas), como nacionais, Ministério da Saúde (MS) e Agência Nacional de Vigilância Sanitária (Anvisa). ${ }^{22}$

O "uso racional" de medicamentos é um assunto discutido em vários encontros de saúde, exemplo destes são, a $39^{\circ}$ Assembleia Mundial de Saúde e a Conferência Mundial sobre o Uso Racional de Medicamentos em 1985 em Nairobi. Na $39^{\circ}$ Assembleia Mundial de Saúde foi criada a Resolução WHA 39.27 (World Health Association), esta preconiza que a prescrição deve ser de acordo com as necessidades clínicas, em doses adequadas às suas particularidades individuais, por período de tempo necessário e com o menor custo para eles e sua comunidade. No Brasil o uso de medicamentos é controlado pela Portaria 3.916/98 do MS, a Política Nacional de Medicamentos, que estabelece bases e diretrizes, e determina prioridades, entre elas a promoção do uso racional de medicamentos, fortalecida pelo Decreto 3.181/99, pela Resolução 391/99 e por decretos posteriores, que regulamentam diversos fatores relativos à implantação da política de medicamentos genéricos no Brasil. ${ }^{23}$

Observa-se que a população que reside nesta região oeste (Figura 2) da cidade vive em situação de vulnerabilidade social, este é um bairro que não apresenta saneamento básico em alguns pontos, faz vizinhança com um deposito de lixo (lixão), algumas ruas não são asfaltadas, as crianças tem acesso à escola, mas muitas passam a maior parte do tempo nas ruas, o modo de sobrevivência de algumas famílias vem da coleta de materiais recicláveis.

A vulnerabilidade social revela-se na violência cotidiana a qual estão submetidas estas famílias na necessidade de buscar o sustento da casa e na falta de atividades que the sejam significativas no contexto familiar e escolar ${ }^{24}$. É importante ressaltar que o serviço em estudo não é o único serviço especializado para atender pessoas com sofrimento 
psíquico, pois o município conta também com os atendimentos nos CAPs e em três hospitais locais que também ofertam atendimentos psiquiátricos no município, porém, estes serviços não foram objeto da pesquisa.

Frente a estes dados observa-se a necessidade da criação de estratégias de atenção a saúde mental, visa-se a importância da implantação e implementação de serviços de saúde mental comunitários especializados na atenção a adolescência. Os estudos epidemiológicos em saúde mental no Brasil devem ser estimulados, pois suas informações caracterizam as pessoas em seus próprios ambientes, o que amplia a visão médica tradicional, mais concentrada em hospitais que na comunidade.

O aumento de estudos nesta área poderá indicar quadros psicopatológicos e fatores associados referentes a cada região e cultura. A partir do conhecimento, pode-se desenvolver e implantar serviços comunitários em saúde mental de forma que o desenvolvimento dos serviços sustente-se, que este atenda as necessidades da região a qual esta implantada, considerando as características específicas da região e do contexto social brasileiro.

\section{Considerações Finais}

O estudo revelou significativos apontamentos para o delineamento de atenção a saúde no que se refere as pessoas com transtornos psíquicos, tanto para as mulheres adultas jovens, como para as demais pessoas identificadas no estudo (adolescentes, homens e idosos). Pode-se identificar as necessidades da implantação de Políticas Públicas voltadas para atenção a saúde mental na região oeste do município de Santa Maria/RS, pois a partir do estudo realizado observou-se que a maioria dos usuários correspondem a esta região administrativa.

Sugere-se que outros estudos desta natureza sejam realizados, no sentido de possibilitar o reconhecimento do contexto da atenção em saúde mental dos municípios, uma vez que a caracterização dessa demanda viabiliza o reconhecimento de possíveis entraves existentes na infraestrutura e processo de trabalho dos serviços do Sistema Único de Saúde, que comprometem o cuidado em saúde. Esse reconhecimento, em adição, contribuirá para a elaboração de políticas públicas que visem a uma atenção de melhor qualidade, empoderando os serviços já existentes no território.

\section{Referências Bibliográficas}

1. SILVA DS, AZEVEDO DM. A reforma psiquiátrica na visão de quem cuida: percepções de profissionais do serviço residencial terapêutico. Esc. Anna Nery. 2011. vol.15, n.3, pp. 587-594.

2. Kantorski LP, Coimbra VCC, Demarco DA, Eslabão AD, Nunes CK, Guedes AC. A importância das atividades de suporte terapêutico para o cuidado em um Centro de atenção. Journal of Nursing and Health. 2011; Vol. 1, No 1.

3. Ballarin MLGS, Carvalho FB, Ferigato SH. Os diferentes sentidos do cuidado: considerações sobre a atenção em saúde mental. O Mundo da Saúde, São Paulo: 2010; 34(4):444-450.

4. Nunes M, Juca VJ, Valentim CPB. Ações de saúde mental no Programa Saúde da Família: confluências e dissonâncias das práticas com os princípios das reformas psiquiátrica e sanitária. Cad. Saúde Pública. 2007; vol.23, n.10.

5. Gomes AMA, Um olhar sobre depressão e religião numa perspectiva compreensiva. Estudos de Religião. 2011; v. 25, n. 40, 81-109.

6.ONOCKO-CAMPOS RT, FURTADO JP. Entre a saúde coletiva e a saúde mental: um instrumental metodológico para avaliação da rede de Centros de Atenção Psicossocial (CAPS) do Sistema Único de Saúde. Cad. Saúde Pública. 2006; vol.22, n.5, pp. 1053-1062.

7. Pegoraro RF, Caldana RHL. Mulheres, loucura e cuidado: a condição da mulher na provisão e demanda por cuidados em saúde mental. Saúde soc. 2008; vol.17, n.2.

8. Resende MC, Almeida CP, Favoreto D, Miranda EG, Silva GP, Vicente JFP, Queiroz LA, Duarte PF, Galicioli SCP. Saúde mental e envelhecimento. 2011; Vol. 42, No 1.

9. Papalia DE, Olds SW. Desenvolvimento humano. Tradução de Daniel Bueno. 12. ed. Porto Alegre : Artmed, 2008. 10. Organização Mundial de Saúde. CID-10: Classificação Estatística Internacional de Doenças e Problemas Relacionados á Saúde/ Organização Mundial da Saúde. São Paulo, SP: EDUSP, 2008.

11. Siegel S, CastellanNJJr. Estatística não-paramétrica para ciências do comportamento. Porto Alegre, RS: Artmed, 2006. 
12. Campos H. Estatística experimental não-paramétrica. $4^{a}$ ed., Piracicaba, 1983.

13. Andrade LHSG, Viana AC, Silveira CM. Epidemiologia dos transtornos psiquiátricos na mulher. Rev. Psiq. Clín, v. 33, n. 2, p. 43-54, 2006.

14. Anselmi L, Barros FC, Minten GC, Glgante DP, Horta BL, VIctora CG. Prevalência e determinantes precoces dos transtornos mentais comuns na coorte de nascimentos de 1982, Pelotas, RS. Rev Saúde Pública;42(Supl. 2), 2008.

15. Cardoso L, Galera SAF. Internação psiquiátrica e a manutenção do tratamento extra-hospitalar. Rev Esc Enferm USP; 45(1):87-94. 2011.

16. Davim RMB, Germano RM, Menezes RMV, Carlos DJD. Adolescente/adolescência: revisão teórica sobre uma fase crítica da vida. REVRENE. V.10, n. 2. 2009.

17. Salles MM, Barros S. Vida cotidiana após adoecimento mental: desafio para atenção em saúde mental. Acta Paul Enferm. 22(1):11-6. 2009.

18. Lopes CS, Faerstein E, Chor D. Eventos de vida produtores de estresse e transtornos mentais comuns: resultados do Estudo Pró-Saúde. Cad. Saúde Pública, Rio de Janeiro, 19(6), 2003.

19. Queiroz VDC. A saúde mental na atenção primária. Serviço Social \& Realidade, Franca, v. 19, n.1, p. 125-152, 2010. 20. Junior ARS, Souza MC. Avaliação do comprometimento funcional na esquizofrenia. Rev. Psiq. Clín. v. 34, n.2, p. 164168, 2007.

21. Gonçalves DM, Kapczinski F. Transtornos mentais e comunidade atendida pelo programa saúde da família. Cad. Saúde Pública. Rio de Janeiro, v. 24, n.7, p. 1641-1650, 2008.

22. Brasil. Resolução $n^{0} 510$ de 1 de outubro de 1999. Disponível em: http://www.anvisa.gov.br/hotsite/genericos/legis/ resolucoes/510_99.htm acessada em 23/12/2013.

23. Wanderley VE, Maia JA, Vilela, RQB. A Prescrição Medicamentosa Ambulatorial no Internato: Formação e Prática. Revista Brasileira de Educação Médica 34 (2), 2010.

24. Guedes PCW, Marques TB, D’Assumção CF, Silva MA, Barbosa LNF. Representação social, ansiedade e depressão em adolescentes puérperas. Rev. SBPH. vol.15, n.1, pp. 194-211. 2012.

\section{Aline Sarturi Ponte}

Endereço para correspondência - Venâncio Aires, 1638, apto 4. Bairro Bom fim, CEP 97010-002, Santa Maria, RS, Brasil.

E-mail: alinesarturi@hotmail.com

Lattes: http://lattes.cnpq.br/8576659601127889

Ana Cristina Von Bock Bolli - anabolli@hotmail.com

Maria Saleti Lock Vogt - saletevogt@gmail.com

Miriam Cabrera Corvelo Delboni - miriamdelboni@gmail.com

Rosimara Adilia Piovezan - rosi.piovezan@hotmail.com

\section{Enviado em 03 de junho de 2014.}

Publicado em 09 de outubro de 2014. 